REVISTA DE CIENCIAS AGRÍCOLAS

Volumen 31 (1) : 92 - 105

Segundo Semestre

ISSN Impreso 0120-0135

\title{
EVALUACIÓN DE AISLAMIENTOS NATIVOS DE Beauveria spp. SOBRE Tecia solanivora (LEPIDOPTERA: GELECHIIDAE) IN VITRO
}

\section{IN VITRO EVALUATION OF NATIVE Beauveria spp. ISOLATES AGAINST Tecia solanivora (LEPIDOPTERA: GELECHIIDAE)}

\author{
Jorge Enrique Villamil C. ${ }^{1}$, John Wilson Martínez O. ${ }^{2}$
}

Fecha de recepción: Abril 21 de 2014

Fecha de aceptación: Junio 03 de 2014

\section{RESUMEN}

Se evaluó la eficacia de hongos entomopatógenos nativos en el biocontrol de Tecia solanivora (Lepidoptera: Gelechiidae) in vitro. Los aislamientos fueron obtenidos de larvas y adultos de gusano blanco Premmotrypes vorax, barrenador del curubo Aepytus sp, picudo de la palma Rinchophorus palmarum, broca del café Hypothenemus hampei, polilla guatemalteca Tecia solanivora y palomilla de la papa Phthorimaea operculella infectados, de los municipios de Ventaquemada, Umbita, Coper (Boyacá) y San gil (Santander). El trabajo se realizó en el laboratorio de control biológico del Grupo Manejo Biológico de Cultivos en Tunja, Colombia. Se seleccionaron in vitro, de forma preliminar cinco de los siete aislamientos de hongos obtenidos: Bv01, Bv03, Bv04, Bv05 y Bv07, los cuales ocasionaron mortalidades superiores al 10\%. De acuerdo con la caracterización morfológica realizada, los hongos encontrados pertenecen al género Beauveria. La evaluación de su eficacia se realizó con 10 larvas de T. solanivora desinfectadas y sumergidas en suspensión de 1x107esporas. $\mathrm{mL}^{-1}$, las cuales se observaron durante 32 días, registrando cada 8 días la mortalidad. Se empleó un diseño completamente al azar, con 6 tratamientos, 4 repeticiones y una prueba de separación de medias de Tukey. Los resultados mostraron que 32 días después de la inoculación los aislamientos Bv03 y Bv05 fueron los que produjeron la mayor mortalidad acumulada (7,4 y 8,5\%, respectivamente). Se confirma el bajo potencial de los hongos entomopatógenos nativos para el control de larvas de T. solanivora.

Palabras clave: Polilla guatemalteca, control biológico, hongos entomopatógenos, aislamientos nativos.

1 Joven investigador COLCIENCIAS-Uptc. I.A., Grupo Manejo Biológico de Cultivos. Universidad Pedagógica y Tecnológica de Colombia. Tunja, Boyacá. Colombia. cavitosa@yahoo.com.

2 Profesor Asistente, I.A., M.Sc. Entomología. Universidad Pedagógica y Tecnológica de Colombia. Tunja, Boyacá. Colombia. john. martinez@uptc.edu.co 


\begin{abstract}
The efficacy of native entomopathogenic fungi for biocontrol of Tecia solanivora (Lepidoptera: Gelechiidae) was evaluated in vitro. Native isolates were obtained from infected Premnotrypes vorax, Aepytus sp., Rhinchophorus palmarum, Tecia solanivora, and Pthorimaea operculella larvae and adults, from the localities of Ventaquemada and Umbita (Boyacá) and San Gil (Santander). This work was developed at the Biological Control Laboratory of the Biological Culture Management Group, Tunja, Colombia. Preliminarily, five of seven isolates, which produced higher than $10 \%$ mortalities, were selected in vitro: Bv01, Bv03, Bv04, Bv05, and Bv07. According to morphological characterization, the isolates belonged to the Beauveria genus. Efficacy was evaluated on $10 \mathrm{~T}$. solanivora larvae, disinfected and submerged in a suspension of $1 \times 10^{7}$ spores. $\mathrm{mL}^{-1}$. Mortality was quantified every 8 days during 32 days. A completely randomized experimental design was used, with 6 treatments and 4 replicates. Tukey's probe was used. Results showed that 32 days after treatment application, isolates Bv03 and Bv05 produced the greatest accumulative mortality percentages (7.4 and $8.5 \%$, respectively). We confirm low native fungi pathogenicity against $T$. solanivora.
\end{abstract}

Key words: Guatemalan moth, biological control, entomopathogenic fungi, native isolates.

\section{INTRODUCCIÓN}

En Colombia el cultivo de la papa integra especialmente a pequeños productores quienes tienen este renglón como su principal fuente de ingresos, bienestar y calidad de vida (Cotes et al., 2003). En la región Cundiboyacense se siembra como monocultivo favoreciendo la presencia continua de plagas y enfermedades que obliga a un manejo fitosanitario exigente y permanente, lo cual genera impacto ambiental negativo por el uso de agroquímicos y remoción de suelo en zonas de ladera y/o páramo alto, lo que finalmente se ve reflejado en altos costos de producción (Cotes et al., 2003; Porras, 2010).

Dentro de los insectos plaga que afectan éste cultivo, se destaca Tecia solanivora (Lepidóptera: Gelechiidae) por su amplia distribución. Esta es actualmente la plaga de mayor relevancia en Centro y Suramérica, convirtiéndose en la plaga de mayor importancia económica en las zonas productoras de papa en Colom- bia. Las condiciones ambientales en las que se desarrolla el cultivo, junto con la ausencia de mecanismos propios de regulación como enemigos naturales, el uso inapropiado de productos químicos y la falta de implementación de adecuadas prácticas culturales en el manejo del cultivo, favorecen el desarrollo de este insecto (Niño et al., 2004). El control se hace difícil, debido a que el daño que ocasiona durante su ingreso y establecimiento en lotes de cultivo y almacenes es muchas veces imperceptible. Esta plaga en estado larval penetra el tubérculo por un agujero muy pequeño y en su interior se alimenta, forma galerías, deposita sus excrementos, saliendo sólo a empupar cuando ya los tubérculos están severamente afectados, ocasionando pérdidas en campo y almacenamiento hasta del 100\% (Arias et al., 1996).

La investigación sobre la polilla guatemalteca de la papa se ha orientado al estudio de barreras físicas, dinámica de poblaciones, uso de virus, atrayentes químicos y validación del MIP para su control (Calvache, 1985; Yabar, 
1988; Calvache, 1991; Torres et al., 2004; Espitia, 2010). No obstante, la medida más utilizada por los agricultores es el uso de insecticidas químicos como carbamatos, piretroides y organofosforados que representan uno de los mayores costos directos del cultivo, los cuales no siempre son efectivos, incrementando así los costos totales de producción (Mena et al., 2003; Torres et al., 2004; Vergara, 2004). En la mayoría de los casos, los insecticidas son aplicados sin justificación técnica y sólo con el criterio de proteger la cosecha contra el eventual ataque de la plaga (López, 2003).

Ante ésta situación ha surgido la necesidad de desarrollar alternativas de control que aseguren el manejo sostenible y eficiente de la plaga a través del uso de organismos entomopatógenos como hongos, bacterias, nematodos y virus. En este sentido, se han concretado valiosos trabajos de investigación en condiciones de laboratorio, los cuales han reportado alta efectividad en la regulación de poblaciones de la plaga (Espitia, 2010).

Las investigaciones referentes al empleo de hongos entomopatógenos para el biocontrol de T. solanivora son escasas; no obstante, se reportan estudios in vitro y en campo que evalúan Beauveria bassiana y Metarhizium anisopliae sobre huevos y larvas de este insecto plaga, aunque con resultados poco promisorios por su baja eficacia entomopatógena (Feris et al., 2002). La baja eficacia de biocontrol es debida probablemente a la complejidad de la relación biocontrolador-plaga y la influencia que las condiciones ambientales tienen sobre este complejo (Feris, 1999; Feris et al., 2002; Quintero et al., 2010).

Los géneros Beauveria y Metarhizium han evidenciado buenos resultados en el control de otras plagas tropicales (Reyes et al., 1995; France et al., 1999; Nankinga y Moore, 2000; Godonou et al., 2000; Giraldo, 2001; Pariona et al., 2007; Góngora, 2008).
En este estudio se recurrió a aislamientos de hongos entomopatógenos regionales, ya que algunas investigaciones recomiendan utilizar aislamientos nativos extraídos del insecto, puesto que existen modificaciones específicas del hongo hacia el hospedero que pueden mejorar su acción patogénica (France et al., 1999), así como de la zona en donde esté presente la plaga a controlar. Con base en lo anterior, el objetivo de esta investigación fue evaluar la eficacia de hongos entomopatógenos nativos en el biocontrol de Tecia solanivora, en condiciones in vitro en Boyacá.

\section{MATERIALES Y MÉTODOS}

El presente trabajo se desarrolló en el Laboratorio de control biológico del Grupo Manejo Biológico de Cultivos- GMBC - del Programa de Ingeniería Agronómica de la Universidad Pedagógica y Tecnológica de Colombia, sede Tunja, ubicado a una altura de $2680 \mathrm{~m}$, con temperatura promedio de $13^{\circ} \mathrm{C}$.

Los hongos fueron obtenidos de larvas y adultos de gusano blanco Premmotrypes vorax, Barrenador del curubo Aepytus sp, picudo de la palma Rinchophorus palmarum, polilla guatemalteca Tecia solanivora y palomilla de la papa Phthorimaea operculella hallados infectados en campo, de los municipios de Ventaquemada y Umbita (Boyacá) y San gil (Santander). El material colectado se ubicó en cámaras húmedas, a una temperatura aproximada de $23^{\circ} \mathrm{C}$, durante 5 a 8 días, hasta observar el crecimiento micelial sobre los adultos o larvas (Mena et al., 2003).

Las pruebas biológicas con los hongos aislados se realizaron con larvas de $T$. solanivora con peso promedio de $0,020 \mathrm{~g}$, colectadas de tubérculos de papa obtenidos en una finca papera del municipio de Siachoque (Boyacá). El material biológico fue puesto en cuarentena 
durante 30 días en suelo estéril recolectado de la misma finca y alimentado con trozos de papa hasta el inicio de las pruebas de preselección y evaluación.

\begin{abstract}
Aislamiento de hongos entomopatógenos. Se hizo siembra directa del micelio que se desarrolló sobre los adultos y larvas de los diferentes insectos colectadas en campo, en cajas petri que contenían medio de cultivo papa-dextrosaagar (PDA), suplementado con cloranfenicol para evitar la contaminación bacteriana (Mena et al., 2003). Las cajas se incubaron a $25^{\circ} \mathrm{C}$ durante diez días. Se realizaron repiques en PDA del borde del crecimiento de la colonia, hasta lograr colonias puras de los aislamientos (Agrios, 1996). A cada uno de los aislamientos obtenidos se le asignó un código compuesto de letras y números.
\end{abstract}

La remoción de las esporas de los cultivos se realizó con Tween 80 al 0,05\%; a partir de esta suspensión se hicieron diluciones seriadas y con la cámara de Newbauer y un microscopio Nikon ${ }^{\circledR}$ se realizó el recuento de propágulos infectivos por mililitro, a fin de calcular la concentración de la suspensión madre (Mena et al., 2003; Lucero et al., 2004).

\section{Activación y preselección de hongos nativos por su eficacia entomopatógenica hacia lar- vas de T. solanivora.}

Se realizó sobre 10 larvas de T. solanivora, las cuales, previo a la inoculación con el entomopatógeno, se desinfectaron con hipoclorito de sodio $(\mathrm{NaClO}, 0,5 \%)$ durante cinco segundos y se lavaron tres veces con agua destilada estéril (ADE) (Feris et al., 2002). La activación de los aislamientos se hizo de acuerdo a la metodología planteada por López (1994) y Feris et al. (2002).

Una vez obtenida la concentración de la suspensión madre para cada uno de los aislamientos nati- vos, se ajustó a la concentración de 1×107esporas. $\mathrm{mL}^{-1}$. Posteriormente, las larvas de T. solanivora se sumergieron en una caja de petri que contenía la suspensión, durante cinco minutos; al cumplir el tiempo de exposición se retiró de estas el exceso de suspensión mediante inversión de la caja petri sobre una toalla de papel seco y estéril.

Con el fin de disminuir la mortalidad por problemas de contaminación, cada una de las larvas de T. solanivora inoculadas se incubó por separado en un vaso plástico semitransparente de $25 \mathrm{~mL}$ de capacidad, previamente desinfectado, con tapa con orificios y el fondo cubierto por $1 \mathrm{~cm}$ de suelo estéril humedecido con ADE a razón de $0,3 \mathrm{~mL} /$ vaso.

Las larvas inoculadas fueron dejadas a temperatura ambiente durante 15 días, protegidas de la luz solar directa y cada 72 horas se registró la mortalidad y simultáneamente se agregó 0,1 mL/vaso de ADE. En este período no se suministró alimento a las larvas, para favorecer la infección del hongo. Terminadas las observaciones se seleccionaron aquellos aislados de hongos nativos que mostraron mortalidades superiores al 10\%. Las larvas muertas se incubaron en cámara húmeda a $23^{\circ} \mathrm{C}$ para corroborar el crecimiento del hongo sobre el insecto.

Evaluación de la patogenicidad de los aislamientos preseleccionados. Se llevó a cabo con los aislamientos nativos de hongos, seleccionados en las pruebas de preselección sobre larvas de T. solanivora. Cada uno de estos aislamientos fue multiplicado durante 15 días en 5 cajas Petri con PDA, incubadas a $25^{\circ} \mathrm{C}$.

Una vez multiplicados los aislamientos nativos, se obtuvo el inóculo, de acuerdo con la metodología explicada anteriormente. Lograda la concentración de las suspensiones madres, se ajustaron todos los aislamientos fúngicos a la concentración $1 \times 10^{7}$ esporas. $\mathrm{mL}^{-1}$. 
Los insectos empleados en las pruebas se desinfectaron con $\mathrm{NaClO}$ 0,5\%, como se explicó anteriormente. Para la inoculación de los hongos nativos se siguió la metodología de López (1994) y Feris et al. (2002), ya explicada.

Se utilizaron cajas plásticas para inocular las larvas con el entomopatógeno, con orificios por todos sus lados y medidas de $8 \mathrm{~cm}$ de ancho $\mathrm{x}$ $10 \mathrm{~cm}$ de largo $\times 6 \mathrm{~cm}$ de alto, que se expusieron a luz ultravioleta durante 30 minutos para su desinfección. Estas cajas contenían 1,5 cm de suelo estéril y 5 porciones de papa de aproximadamente $2 \mathrm{~cm}^{3}$, previamente desinfectadas con hipoclorito de sodio $0,5 \%$, por 3 minutos y lavadas cuatro veces con agua destilada estéril durante 5 minutos en cada ocasión.

La evaluación de mortalidad de las larvas de $T$. solanivora se realizó cada 8 días, durante 1 mes después de la inoculación, mediante observación directa con estereoscopio de luz (Leica). Las observaciones permitieron discriminar la mortalidad total y la mortalidad causada por el hongo; además, durante cada observación se adicionaron $4 \mathrm{~mL}$ de ADE a cada una de los vasos para mantener la humedad relativa requerida por los hogos (Feris et al., 2002).

Las larvas muertas por cada tratamiento se pusieron en cámara húmeda a $23^{\circ} \mathrm{C}$ durante 15 días, para corroborar el crecimiento del hongo sobre el cuerpo larval (Feris et al., 2002).

Se empleó un diseño experimental completamente al azar (DCA), con 6 tratamientos y 4 repeticiones por tratamiento, para un total de 24 unidades experimentales (U.E). Cada repetición estuvo constituida por una población de 10 larvas de T. solanivora con peso promedio de $0,020 \mathrm{~g}$.
Los datos obtenidos en las evaluaciones de mortalidad de larvas de T. solanivora por tratamiento, fueron sometidos análisis de varianza. Además se analizaron los supuestos de normalidad y homocedasticidad mediante la prueba de Shapiro-wilk y Bartlett y se realizó un análisis de varianza y posteriormente se utilizó la prueba de comparación múltiple de Tukey (Gómez y Gómez, 1984), utilizando el paquete estadístico $R$ versión 3.0.2@.

Identificación de los aislamientos de hongos nativos. Se realizó hasta género de acuerdo a las características morfológicas CMI (1979), Samson et al. (1988); Rodríguez y Del Pozo (2003); Cañedo y Ames (2004) y Zimmerman (2007), mediante la utilización de la clave taxonómica propuesta por Egorova (1980) y, Barnet y Hunter (1998). Se utilizó la tabla Munsell para describir el color de la colonia y se determinó el tipo de crecimiento (Carmichael et al., 1980; Kuppers, 1996).

\section{RESULTADOS Y DISCUSIÓN}

Preselección de hongos nativos por su eficacia entomopatógena hacia larvas de $T$. solanivora. El aislamiento nativos que ocasionó el mayor porcentaje de mortalidad en larvas de T. solanivora tratadas fue $\mathrm{Bv} 03$ (15\%), seguido por Bv01, Bv07, Bv05, Bv04 (Tab.1). Estos evidenciaron igualmente la mejor esporulación sobre el cuerpo de las larvas infectadas, 8 días después de permanecer en cámara húmeda a $23^{\circ} \mathrm{C}$ y esporas con mayor adherencia al cuerpo de la larva (Lucero et al., 2004). Los aislamientos Cp02 y St06 fueron los que originaron menor mortalidad. Las diferencias encontradas podrían deberse a la variabilidad natural de los aislamientos (Ferrón, 1967; Evans, 1986; Rehner y Buckley, 2005) y la especificidad hacia $T$. solanivora, que en el caso del aislamiento Bv03 parece ser mayor. 
Tabla 1. Identificación, origen y porcentaje de mortalidad de aislamientos nativos de hongos evaluados en preselección sobre larvas de T. solanivora in vitro

\begin{tabular}{|c|c|c|c|c|}
\hline $\begin{array}{c}\text { Aislamientos } \\
\text { Nativos }\end{array}$ & Origen & Hospedero & $\begin{array}{c}\text { Mortalidad } \\
\quad(\%)\end{array}$ & DS \\
\hline $\mathrm{Bv} 01^{*}$ & $\begin{array}{c}\text { San Gil } \\
\text { (Santander) }\end{array}$ & Picudo palma Rhynchophorus. palmarum & 12 & $\pm 0,8$ \\
\hline Cp02 & $\begin{array}{c}\text { Coper } \\
\text { (Boyacá) }\end{array}$ & Broca café Hypothenemus hampei & 8 & $\pm 0,8$ \\
\hline $\mathrm{Bv} 03^{*}$ & $\begin{array}{l}\text { Ventaquemada } \\
\text { (Boyacá) }\end{array}$ & Polilla Guatemalteca Tecia solanivora & 15 & $\pm 0,8$ \\
\hline $\mathrm{Bv} 04^{*}$ & $\begin{array}{l}\text { Ventaquemada } \\
\text { (Boyacá) }\end{array}$ & Polilla de papa Phthorimaea. operculella & 10 & $\pm 0,8$ \\
\hline $\mathrm{Bv} 05^{*}$ & $\begin{array}{l}\text { Ventaquemada } \\
\text { (Boyacá) }\end{array}$ & Gusano blanco papa Premnotrypes vorax & 10.5 & $\pm 0,3$ \\
\hline St06 & $\begin{array}{l}\text { Soata ( } \\
\text { Boyacá) }\end{array}$ & Gusano blanco papa Premnotrypes vorax & 7 & $\pm 0,8$ \\
\hline $\mathrm{Bv} 07^{*}$ & $\begin{array}{l}\text { Umbita } \\
\text { (Boyacá) }\end{array}$ & Barrenador del curubo Aepytus sp & 11 & $\pm 0,8$ \\
\hline
\end{tabular}

*: Aislamientos preseleccionados para pruebas de evaluación in vitro.

DS: Desvío

Feris et al. (2002) al activar las cepas Beauveria bassiana Bv006 y Metarhizium anisopliae Mt001 en larvas de primer instar de T. solanivora, observaron como porcentaje mínimo de mortalidad de $7,84 \%$ y máximo $15,72 \%$, respectivamente, empleando una concentración de $1 \times 10^{8}$ esporas. $\mathrm{mL}^{-1}$. Lo anterior concuerda con los resultados obtenidos en el presente trabajo.

De acuerdo con los resultados obtenidos los aislamientos seleccionados para las pruebas de evaluación in vitro fueron entonces Bv01, Bv03, Bv04, Bv05, Bv07.

Evaluación de la eficacia entomopatógena de los aislamientos preseleccionados en el biocontrol de $T$. solanivora

Pruebas de patogenicidad. Los cinco aislados nativos evaluados mostraron baja acción entomopatógena, no superando el 10\% de mortalidad, siendo inferiores a los porcentajes obtenidos en las pruebas de preselección (Tab. 2).
Tabla 2. Porcentaje de mortalidad de larvas de $T$. solanivora evaluadas en pruebas de patogenicidad de aislamientos, in vitro

\begin{tabular}{c|c|c|c|c}
\hline \multirow{2}{*}{ Tratamientos } & \multicolumn{4}{|c}{ Días } \\
\cline { 2 - 5 } & $\mathbf{8}$ & $\mathbf{1 6}$ & $\mathbf{2 4}$ & $\mathbf{3 2}$ \\
\hline T1. Bv01 & 0 & 0 & $4,3 \mathrm{bc}$ & $5 \mathrm{c}$ \\
\hline T2. Bv05 & 0 & 0 & $6,5 \mathrm{ab}$ & $8,2 \mathrm{ab}$ \\
\hline T3. Bv07 & 0 & 0 & $4,7 \mathrm{abc}$ & $7 \mathrm{bc}$ \\
\hline T4. Bv03 & 0 & 0 & $7 \mathrm{a}$ & $10 \mathrm{a}$ \\
\hline T5. Bv04 & 0 & 0 & $3,5 \mathrm{c}$ & $6,2 \mathrm{bc}$ \\
\hline T6. Testigo & 0 & 0 & $5,2 \mathrm{abc}$ & $7,7 \mathrm{ab}$ \\
\hline
\end{tabular}

* En las columnas, valores con letras distintas difieren significativamente Bv01-Bv03-Bv04-Bv05-Bv07 ( $\mathrm{P}<0.01)$.

El análisis de varianza, para el número de larvas muertas mostro que se presentaron diferencias significativas entre aislamientos. De acuerdo con la prueba Tukey ( $\mathrm{P} \leq 0.01)$ (Tab. 2), a partir del día 24 el aislado Bv03 presento el mayor porcentaje de mortalidad, siendo estadísticamente diferente a los demás tratamientos. 
En lepidóptera, Zuñiga y Redolfi (1981) reportan éxito entomopatógeno utilizando $B$. bassiana sobre larvas de tercer instar de Spodoptera frugiperda (Lepidoptera: Noctuidae) con porcentajes de infección de 33,33\% a 71,55\% ; entretanto Melisa (2003), señala mortalidades hasta de 100\% inoculando $B$. brongniartii a una concentración de $1 \times 10^{9}$ esporas. $\mathrm{mL}^{-1}$ en larvas de primer instar de $P$. operculella $S$. tangolias y gelechidos que también atacan a los tubérculos de papa.

Es probable que debido a que se empleó material larval homogéneo en cuanto a peso y no en cuanto a estadio larval, la susceptibilidad de estas se exprese de forma diferente para cada etapa de desarrollo independiente del peso de la larva, originando así los menores porcentajes de mortalidad respecto de las pruebas preliminares.

En las pruebas de eficacia entomopatógena, el testigo presentó una tasa acumulada de supervivencia del 93,5\%, lo que demuestra que la práctica de desinfección superficial de las larvas mediante el tratamiento con hipoclorito de sodio no afectó su supervivencia; de la misma forma, este proceso no inhibió los procesos de germinación de las esporas y crecimiento conidial en los tratamientos, lo cual coincidiría con lo observado por González et al. (1993) y Mena et al. (2003) quienes utilizaron este mismo método. Las posibles causas de la mortalidad observada son atribuidas por los autores antes mencionados, a manipulación de las larvas y problemas fisiológicos como inanición. Arrubla et al. (2010) aseguran que el máximo tolerable de mortalidad en el testigo, durante evaluaciones de patogenicidad, por causas distintas a crecimiento de hongos y bacterias contaminantes es $10 \%$, lo cual se logró en este ensayo.

Mortalidad acumulada. Respecto a la mortalidad acumulada (Fig. 1), a los 32 días después de la infección de las larvas, el mejor tratamiento fue Bv03 con 8,5\% de mortalidad, siendo diferente estadísticamente a los demás tratamientos. El mejor efecto patogénico de este aislamiento nativo, se debe muy posiblemente a que existen modificaciones específicas del hongo hacia el hospedero que pueden mejorar su acción patogénica (Evans, 1992; France et al., 1999).

La baja patogenicidad hacia las larvas de T. solanivora puede explicarse en términos de especificidad de los aislamientos (Feris et al., 2002). La literatura indica que los hongos entomopatógenos pertenecientes a la clase Hyphomycetes atacan un amplio rango de hospederos (Feng y Johnson, 1990); sin embargo, existen aislamientos de B. bassiana y B. brongniartii que tienen un estrecho rango de hospederos que los hace relativamente específicos (McCoy et al., 1988). Los hongos entomopatógenos tienen así la capacidad de especializarse en su acción patogénica en los insectos a los cuales se les aplica el control (Valdez y Vélez, 1998).

Se han evaluado distintos aislamientos de cultivos monoespóricos de B. bassiana obtenidos a partir de la broca del café y otros insectos provenientes de Colombia y otros países por sus características de virulencia y su diversidad genética contra $H$. hampei (Bustillo y Posada, 1996; Cruz et al., 2006; Arrubla et al., 2010). Las investigaciones han permitido conocer que no todas las cepas de B. bassiana son genéticamente iguales, ni controlan la broca en la misma proporción (Arrubla et al., 2010).

Beauveria sp es un hongo saprófito facultativo que controla más de 700 especies de artrópodos, lo que explica el gran interés que ha atraído por su potencial como controlador biológico de plagas. Noobstantesu virulenciay especificidad varían considerablemente entre aislamientos (Lecuona et al., 1996), lo cual sugiere una base 


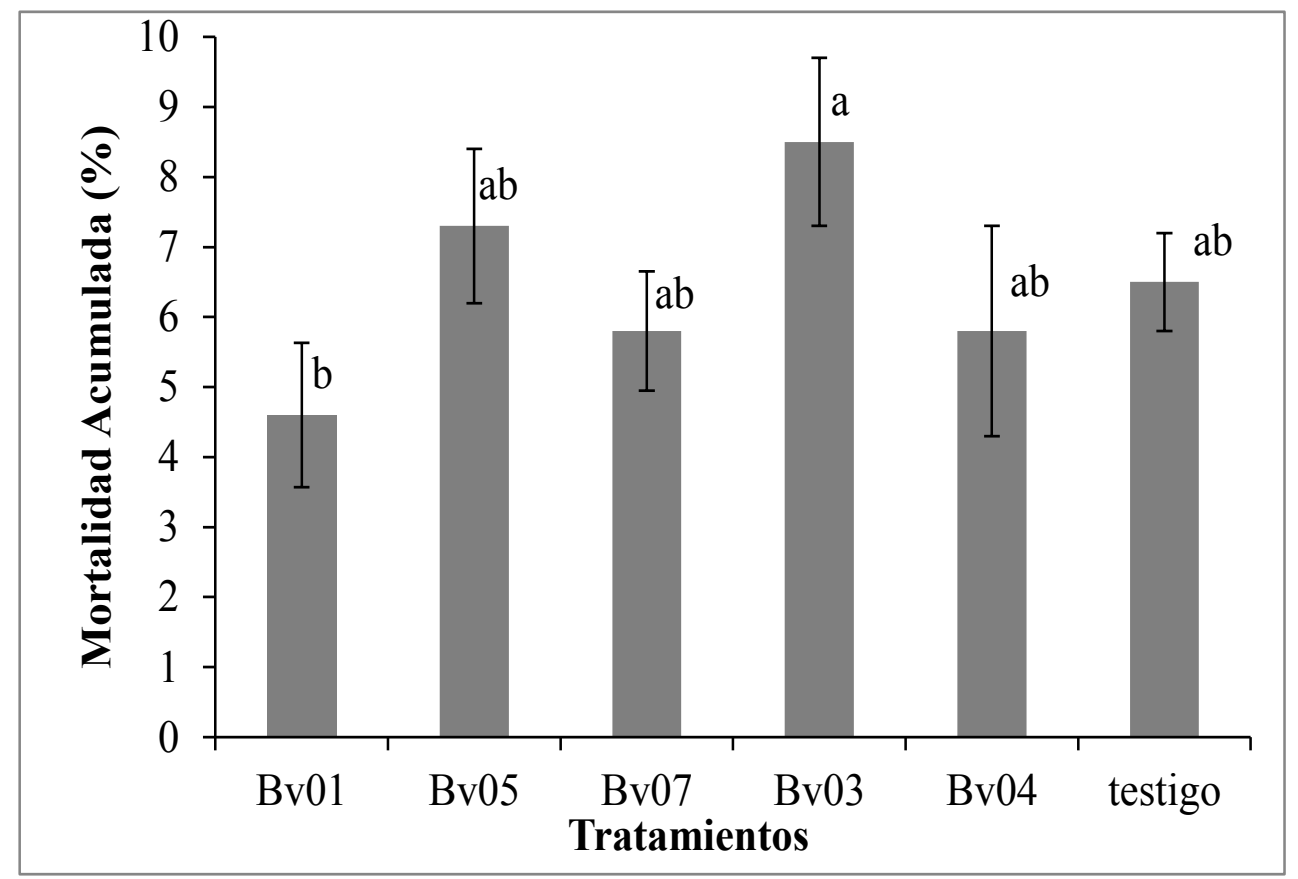

Figura 1. Porcentaje de mortalidad acumulada de los cinco aislamientos de Beauveria spp. sobre larvas de $T$. solanivora $n=4$. Letras distintas indican diferencias estadísticamente significativas según Tukey. Los intervalos sobre cada barra representan la desviación estándar.

genética diferente (Berreta et al., 1998). La existencia de factores abióticos (temperatura, humedad, viento, lluvias) y bióticos (parásitos, depredadores, planta huésped) predisponen al hospedero tanto como al entomopatógeno para el momento de la infección y juegan un papel decisivo para el éxito de la relación patógeno hospedero (Tanada y Kaya, 1993).

\section{Esporulación de los hongos en las larvas} tratadas. El análisis de varianza, para la esporulación de larvas muertas de T. solanivora mostró que existen diferencias significativas entre aislamientos. De acuerdo con la prueba Tukey (Ps 0.01) (Fig. 2), el aislado Bv03 presento el menor tiempo entre la muerte y el cubrimiento micelial, con 7,2 días, para un total de 39,2 días después de la inoculación de las larvas, siendo estadísticamente diferente con la mayoría de los tratamientos.

De acuerdo con Mena et al. (2003) las diferentes etapas de desarrollo de los hongos entomopatógenos en larvas de $P$. vorax, (inoculación a muerte, muerte a cubrimiento micelial, muerte a liberación de conidias), presentaron rangos entre 14 y 20 días. Al respecto González et al. (1993) encontraron que cepas de B. bassiana en el biocontrol de $H$. hampei desarrollaron el total de éstas etapas en un promedio de 8,2 días. Torres y López (1997), en un estudio realizado en laboratorio con $B$. bassiana y larvas de $P$. vorax, observaron que el período de iniciación de los síntomas de ataque del entomopatógeno osciló entre 5 a 7 días, y de ahí a la esporulación, hubo un período de 8 a 13 días, para un total de 20,5 días. El tiempo de muerte a esporulación del asilamiento Bv03 se encontraría dentro de los rangos antes mencionados.

Identificación de aislamientos de hongos nativos. La caracterización macro y microscópica y la identificación de los aislamientos evaluados se presentan en las Tablas 3 y 4 . 


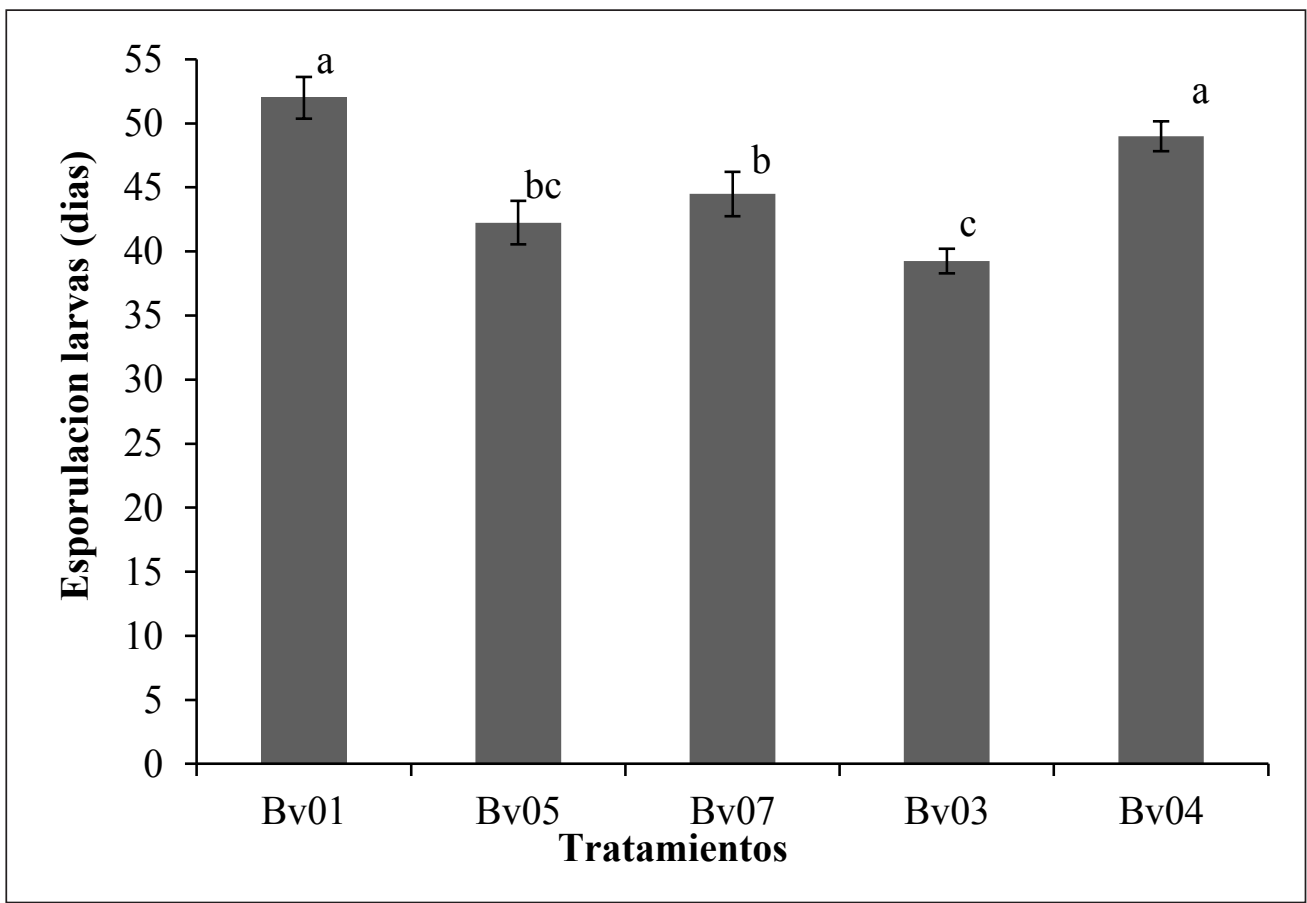

Figura 2. Esporulación de los cinco aislamientos de Beauveria sobre larvas de T. solanivora $n=4$. Letras distintas indican diferencias estadísticamente significativas según Tukey los intervalos sobre cada barra representan la desviación estándar.

Tabla 3. Caracterización macroscópica de los aislamientos de hongos entomopatógenos nativos

\begin{tabular}{c|c|c|c|c|c}
\hline $\begin{array}{c}\text { Hongo } \\
\text { nativo }\end{array}$ & $\begin{array}{c}\text { Crecimiento } \\
\text { de colonias }\end{array}$ & $\begin{array}{c}\text { Crecimiento } \\
\text { completo en } \\
\text { PDA }\end{array}$ & $\begin{array}{c}\text { Aspecto de la } \\
\text { colonia }\end{array}$ & Color & $\begin{array}{c}\text { Superficie } \\
\text { colonia }\end{array}$ \\
\hline Bv01 & $\begin{array}{c}\text { Circular } \\
\text { con bordes } \\
\text { definidos }\end{array}$ & 15 días & polvoriento & $\begin{array}{c}\text { Amarillo } \\
\text { crema }\end{array}$ & plano \\
\hline Bv03 & circular & 17 días & aterciopelado & $\begin{array}{c}\text { amarillo } \\
\text { pálido }\end{array}$ & $\begin{array}{c}\text { semi } \\
\text { elevada }\end{array}$ \\
\hline Bv04 & circular & 16 días & aterciopelado & $\begin{array}{c}\text { amarillo } \\
\text { pálido }\end{array}$ & $\begin{array}{c}\text { semi } \\
\text { elevada }\end{array}$ \\
\hline Bv05 & circular & 15 días & aterciopelado & $\begin{array}{c}\text { blanco } \\
\text { algodonoso }\end{array}$ & $\begin{array}{c}\text { elevada } \\
\text { Bv07 }\end{array}$ \\
\hline circular & 17 días & aterciopelado & $\begin{array}{c}\text { amarillo } \\
\text { pálido }\end{array}$ & semi \\
\hline
\end{tabular}


Tabla 4. Identificación y caracterización microscópica de aislamientos de hongos entomopatógenos nativos

\begin{tabular}{c|c|l|l|l|l|l}
\hline $\begin{array}{c}\text { Hongo } \\
\text { nativo }\end{array}$ & conidióforos & \multicolumn{1}{|c|}{ conidias } & fiálides & raquis & $\begin{array}{l}\text { Tamaño } \\
\text { conidia }\end{array}$ & Genero \\
\hline Bv01 & $\begin{array}{c}\text { simples e } \\
\text { irregulares }\end{array}$ & $\begin{array}{l}\text { hialinas, lisas y } \\
\text { globosas }\end{array}$ & globosa & largo & pequeña & Beauveria \\
\hline Bv03 & Sencillos & $\begin{array}{l}\text { hialinas, lisas y } \\
\text { elipsoidales }\end{array}$ & subglobosas & largo & grande & Beauveria \\
\hline Bv04 & Sencillos & $\begin{array}{l}\text { hialinas, lisas y } \\
\text { elipsoidales }\end{array}$ & subglobosas & largo & grande & Beauveria \\
\hline Bv05 & Sencillos & $\begin{array}{l}\text { hialinas, lisas y } \\
\text { elipsoidales }\end{array}$ & subglobosas & largo & grande & Beauveria \\
\hline Bv07 & Sencillos & $\begin{array}{l}\text { hialinas, lisas y } \\
\text { elipsoidales }\end{array}$ & subglobosas & largo & grande & Beauveria \\
\hline
\end{tabular}

De los 5 aislados nativos caracterizados morfológicamente como Beauveria spp., uno se obtuvo a partir de $T$. solanivora en forma natural (Feris, 1999; Feris et al., 2002) y cuatro de larvas y adultos de especies de insectos que causan daño en diferentes cultivos. Las diferencias en los resultados obtenidos en las evaluaciones preliminares y posteriores de los aislamientos evaluados pueden deberse a que las cepas de $B$. bassiana no son genéticamente iguales, ni controlan su blanco en la misma proporción, ya que existe una alta especificidad de este hacia un huésped, pese a no ser aislado de su hospedero natural (Zuñiga y Redolfi, 1981; Feng y Johnson, 1990; Luz et al., 1998; Feris, 1999; Melisa, 2003).

\section{CONCLUSIONES}

Los aislamientos nativos del género Beauveria no presentaron una alta eficacia entomopatógena sobre larvas de T. solanivora en condiciones in vitro, por lo cual no podrían ser validadas como herramienta para el manejo integrado del insecto-plaga en condiciones de campo.

Entre los aislamientos de Beauveria encontrados, el denominado Bv03, ocasionó la mayor mortalidad y evidenció el menor tiempo para su esporulación en larvas de T. solanivora.

Existe una diversidad de hongos del genero Beauveria que podrían ser empleados en evaluaciones para el biocontrol de otras especies de insectos plaga de importancia económica en Boyacá y Santander.

Frente al panorama de la baja eficacia de los hongos entomopatógenos sobre $T$. solanivora, la investigación se podría enfocar hacia el uso de otros biocontroladores como virus, con éxito en el tratamiento de semilla, o nematodos que pueden tener posibilidades en la implementación en almacén y campo de programas para el manejo integrado de este insecto plaga de gran importancia económica en Colombia.

\section{AGRADECIMIENTOS}

A COLCIENCIAS en el Programa Jóvenes Investigadores e Innovadores 2012, la Universidad Pedagógica y Tecnológica de Colombia U.P.T.C. (Tunja) y la Corporación Centro de Innovación del Trópico Alto sostenible CEI3TAS por la cofinanciación de este proyecto. Al I.A 
Raúl Tenza Cardenal por sus valiosos aportes y colaboración, y al equipo de trabajo del Laboratorio de control biológico y Grupo de Manejo de Cultivos GMBC.

\section{BIBLIOGRAFÍA}

AGRIOS, G. 1996. Fitopatología. Edición Limusa, México D.C. 838 p.

ARIAS, J., JARAMILLO, J., ARÉVALO, E., ROCHA, N. y MUÑOZ, G.1996. Evaluación de la incidencia y severidad del daño de Tecia solanivora en papa en el departamento de Antioquia. Boletín técnico. (Corpoica, ICA, Ministerio de Agricultura y desarrollo rural). Medellín, Colombia. 24p.

ARRUBLA, P., CÁRDENAS, R. y POSADA, F. 2010. Efecto de la humedad relativa sobre la germinación de las esporas de Beauveria bassiana y la patogenicidad a la broca del café Hypothenemus hampei. Revista U.D.C.A Actualidad \& Divulgación Científica. 13(1):67-76.

BARNETT, H. y HUNTER, B. 1998. Illustrate genera of imperfect fungi. Fourth edition. The American Phytopathological Society. Minnesota. 217p.

BERRETA, M., LECUONA, R., ZANDOMENI, R. y GRAU, O. 1998. Genotyping isolates of the entomopathigenic fungus Beauveria bassiana by RAPD with fluorescent labels. Journal of Invertebrate Pathology. 71(2):145 - 150.

BUSTILLO, A. y POSADA, F. 1996. El uso de entompatógenos en el control de la broca del café en Colombia. Revista Manejo Integrado de plagas de Costa rica. 42: 1-13.

CARMICHAEL, J., KENDRICK, W., CONNERS, I. y SIGLER, L. 1980. Genera of Hyphomycetes. University of Alberta press. Ed. Edmonton, $386 \mathrm{p}$.
CAÑEDO, V. Y AMES, T. 2004. Manual de Laboratorio para el Manejo de Hongos Entomopatógenos. Editorial Centro Internacional de la Papa (CIP), Perú, 62 p.

CALVACHE, H. 1985. El gusano blanco de la papa Premnotrypes vorax (Hustache) y su control. Revista Nueva Agricultura Tropical. 36(3):3 - 7 .

CALVACHE, H. 1991. Efecto de barreras vegetales y químicas en el control del gusano blanco de la papa (Premnotrypes vorax Hustache). Revista Latinoamericana de la Papa. 4(1):22 - 35.

COMMONWEALTH MYCOLOGICAL INSTITUTE. (CMI). 1979. Descriptions of Pathogenic Fungi and Bacteria CMI, Beauveria bassiana, No. 602.

COTES, A., ZULUAGA, M., ESPINEL, C., VILLAMIZAR, L., TORRES, L., GÓMEZ, M. y LÓPEZ, J. 2003. Evaluación en campo de un Bioplaguicida a base de Beauveria bassiana para el control biológico del gusano blanco de la papa (Premnotrypes vorax) en la región Cundiboyacense y transferencia de la tecnología a los agricultores. Edición Produmedios Corpoica, Colombia, $20 \mathrm{p}$.

CRUZ, L., GAITÁN, A. y GÓNGORA, B. 2006. Exploiting the genetic diversity of $B$. bassiana for improving the biological control of the coffee berry borer through the use of strain mixtures. Applied Microbiology and Biotechnology. 71(6):918 - 926.

ESPITIA, E. 2010. Manejo Integrado de Insectos Plaga del cultivo de la Papa. p 1-132. En: Simposio Nacional de Actualización en el Cultivo de Papa Solanum tuberosum L. Tunja, Colombia.

EVANS, H. 1986. Uso actual y potencial de los hongos entomopatógenos para el 
control biológico de artrópodos plagas. Revista Miscelánea sociedad Colombiana de Entomología. 21:9 - 14.

EVANS, H. 1992. Uso actual y potencial de los hongos entomopatógenos para el biocontrol de artrópodos plaga. Revista Miscelánea sociedad Colombiana de Entomología. 21:9 - 4.

EGOROVA, L. 1980. Hongos del suelo del Lejano Este, Hyphomycetes. Editorial Ciencia, Leningrado, $27 \mathrm{p}$.

FERIS, M. 1999. Evaluación en laboratorio de la actividad biocontroladora de cepas de Beauveria bassiana y Metarhizium anisopliae sobre Tecia solanivora (Povolny). Tesis de grado Ingeniero Agrónomo, Facultad de Agronomía, Universidad Nacional. Bogotá, D.C. 100 p.

FERIS, M., GUTIÉRREZ, C., VARELA, A. y ESPITIA, E. 2002. Evaluación de los hongos entomopatógenos Beauveria bassiana y Metarhizium anisopliae sobre Tecia solanivora (Lepidoptera: Gelechiidae). Revista Colombiana de Entomología. 28(2):179-182.

FERRÓN, P. 1967. Estudio en condiciones de laboratorio del desarrollo ecológico favorable del hongo Beauveria tenella. Revista francés. 12:257 - 293.

FENG, M. y JOHNSON, J. 1990. Relative virulence of six isolates of Beauveria bassiana on Diuraphis noxia (Homoptera: Aphididae). Review Environmental Entomology. 19(3):785 - 790.

FRANCE, A., GERDING, A., SANDOVAL, S., ESPINOSA, Y., y VIVANCO, E. 1999. Patología de Insectos. Edición INIA, Quilamapu, Chile. $119 \mathrm{p}$.

GIRALDO, E. 2001. Actividad lipolítica y proteolítica de Beauveria bassiana y Metarhizium anisopliae y su relación con la patogenicidad sobre Hypotenemus hampei (Coleoptera: Scolytidae). Revista Colombiana de Entomología. 27(1-2):61 - 65 .

GÓNGORA, C. 2008. Los hongos entomopatógenos en el control de insectos, pp. 133-149. En: Bustillo, P. Los insectos y su manejo en la caficultura Colombiana. Edición Cenicafé, Caldas, $149 \mathrm{p}$.

GONZÁLEZ, M., POSADA, F. y BUSTILLO, A. 1993. Bioensayo para evaluar la patogenicidad de Beauveria bassiana (Bals.) Vuill. Sobre la Broca del Café, Hypothenemus hampei (Ferrari). Revista Colombiana de Entomología. 19(4):123 - 130.

GODONOU, K., GREEN, K., ODURO, K., LOMER, C. y AFREH, K. 2000. Field Evaluation of selected formulations of Beauveria bassiana for the management of the banana weevil Cosmopolites sordidus on plantain (Musa spp., AAB Group). Review Biocontrol Science and Technology. 10(1):779 - 778.

GÓMEZ, K. y GÓMEZ, A. 1984. Statistical procedures for agricultural research. Edition John Wiley and Sons, New York. 680 p.

KUPPERS, H. 1996. Atlas de los colores. Editorial Blume, Barcelona. $69 \mathrm{p}$.

LECUONA, R., TIGANO, M. y DÍAZ, B. 1996. Characterization and pathogenicity of Beauveria bassiana against Diatraea saccharalis (F.) (Lepidoptera: Pyralidae) in Argentina. Annals Sociedade Entomologica do Brasil. 25:299 - 307.

LÓPEZ, A. 2003. Generación y transferencia de tecnología para el control biológico del gusano blanco Premnotrypes vorax y la polilla guatemalteca Tecia solanivora, plaga de importancia económica en el cultivo de la papa en Colombia. p. 184-186. En: Memorias II Taller Nacional Tecia solanivora: "Presente y futuro de la investigación en Colombia sobre polilla guatemalteca". Bogotá, Colombia. 
LÓPEZ, J.C. 1994. Efecto patogénico de cuatro aislamientos de Beauveria bassiana (Blas. Viull. (Hyphomycetos) sobre larvas de Bombyx mori L. (Lepidoptera: Bombycidae) en laboratorio. Revista Colombiana de Entomología. 20(1):53 - 60.

LUCERO, A., PEÑA, L. y BACCA, T. 2004. Evaluación de actividad biocontroladora de Beauveria bassiana y Metarhizium anisopliae sobre larvas de Ancognatha scarabaeiodes (Coleoptera: Scarabaeidae). Revista Ciencia \& Tecnología Agropecuaria CORPOICA. 5(1):43 - 48.

LUZ, C., TIGANO, M., SILVA, L., CORDEIRO, C. y ALJANABI, S. 1998. Selection of Beauveria bassiana and Metarhizium anisopliae isolates to control Triatoma infestans. Review Memoria do Instituto Oswaldo Cruz. 93(6):839 - 846.

MCCOY, C., SAMSON, R. y BOUCIAS, D. 1988. Entomogenous fungi. Review Bioscience. 5:151-236. To cited for: Brian, F., Maddos, J. 1996. Especificity in microbe insect interactions. Review Bioscience. 46(6):410 - 421.

MENA, J., PEÑA, L. y GONZÁLEZ, R. 2003. CL50 y variación de la patogenicidad en aislamientos de Beauveria bassiana y Metarhizium anisopliae evaluados en poblaciones de Premnotrypes vorax. Revista Innovación y Cambio Tecnológico de CORPOICA. 4(1):49 - 54.

MELISA, V. 2003. Caracterización de 3 cepas de B. brongniartii (Saccardo) Petch y su virulencia en Phthorimaea operculella (Zeller) y Symmetrischema tangolias (Gyen). Tesis pregrado Biólogo, Facultad de Ciencias Biológicas, Universidad de San Marcos. Lima.177 p.

NANKINGA, C. y MOORE, D. 2000. Reduction of banana weevil populations using diferent formulations of the entomopathogenic fungus Beauveria bassiana. Review Biocontrol Science and Technology. 10(1):645 - 657.
NIÑO, L., ACEVEDO, E., BECERRA, F. y GUERRERO, M. 2004. Aspectos de la biología y fluctuación poblacional del gusano blanco de la papa Premnotrypes vorax Hustache (Coleoptera: Curculionidae) en Mucuchíes, estado de Mérida, Venezuela. Revista Entomotrópica. 19(1):15 - 19.

PARIONA, N., CASTELLANOS, P. y LEÓN, E. 2007. Capacidad entomocida de cepas nativas de Beauveria sp., sobre Schistocerca piceifrons peruviana (Lynch Arribalzaga, 1903). Revista Biología de Perú. 14(2):253 - 257.

PORRAS, P. 2010. Manejo Integrado del Cultivo de la Papa. p 1-45. En: Simposio nacional de actualización en el cultivo de papa Solanum tuberosum L. Tunja, Colombia.

QUINTERO, I., RIVERA, L., GONZÁLEZ, E., CAMACHO, B., MEZA, N., MONTERO, F. y GUDIÑO, S. 2010. Experiencias del uso de entomopatógenos para el control de la polilla guatemalteca. p 40-42. En: Boletín Informativo Instituto de Investigaciones Agrícolas del Estado Trujillo. Venezuela.

REYES, I., BUSTILLO, A. y CHAVES, B. 1995. Efecto de Beauveria bassiana y Metarhizium anisopliae sobre el parasitoide del café Cephalonomia stephanoderis. Revista Colombiana de Entomología. 21(4):199 - 204.

RODRÍGUEZ, A. y DEL POZO, E. 2003. Aislamiento de hongos entomopatógenos y evaluación de su virulencia sobre Trialeurodes vaporariorum (West.). Revista Agro ciencia. 7(2):71 - 78.

REHNER, S. y BUCKLEY, E. 2005. A Beauveria phylogeny inferred from nuclear ITS and EF-1a sequences: evidence for cryptic diversification and links to Cordyceps teleomorphs. Review Mycologia. 97:84 - 98. 
SAMSON, R., EVANS, H. y LATGE, J. 1988. Atlas of Entomopathogenic Fungi. Edition Springer-Verlag, Berlín. 300 p.

TANADA, Y. y KAYA, H. 1993. Insect Pathology. Academic press, Inc. United estates of America. $366 \mathrm{p}$.

TORRES, L., ESPINEL, C., VILLAMIZAR, L., GÓMEZ, M., ZULUAGA, M., LÓPEZ, J., COTES, A. y LÓPEZ, A. 2004. Desarrollo de un insecticida microbiano para el control biológico del gusano blanco de la papa. Boletín Técnico. Edición Produmedios de CORPOICA, Colombia. $75 \mathrm{p}$.

TORRES, P. y LÓPEZ, A. 1997. Estudios Básicos sobre el control microbiológico del gusano blanco de la papa Premnotrypes vorax con Beauveria spp. y Metarhizium sp. Revista Colombiana de Entomología. 23(1-2):83-88.

VALDEZ, B. y VÉLEZ, P. 1998. Caracterización bioquímica cualitativa de aislamientos de Beauveria bassiana de una colección de hongos de CENICAFÉ. Revista Colombiana de Entomología. 24(1-2):61 - 66.

VERGARA, R. 2004. Enfoque agroecológico del empleo de entomopatógenos para el control de plagas. p 34. En: VIII Seminario de Agroecología de Agromedicina y Medio Ambiente. Tunja, Colombia.

YABAR, L. 1988. Integración de prácticas culturales para el control del gorgojo de los Andes Premnotrypes spp. Revista Latinoamericana de la Papa. 1(1): 120-131.

ZIMMERMAN, G. 2007. Review on safety of the entomopathogenic fungus Beauveria bassia$n a$ and Beauveria brongniartii. Review Biocontrol Science and Technology. 7(5-6):553-596.
ZUÑIGA, D. y REDOLFI, L. 1981. Infección de Beauveria bassiana (Bals) (Deuteromycetes: moniliales) sobre Spodoptera frugiperda (Smith) en laboratorio. Revista Peruana de Entomología. 24(1):103 - 106. 